\title{
Efficacy of Tadalafil on Ureteral Stent Symptoms: A Randomized Controlled Trial
}

\author{
Alireza Farshi Haghro ${ }^{1,{ }^{*}}$, Sakineh Hajebrahimi ${ }^{1,{ }^{* *}}$, Abbas Jabbari ${ }^{1}$, Homayoun Sadeghi ${ }^{2}$ and Hossein \\ Babaei $^{3}$ \\ ${ }^{1}$ Urology Department, Tabriz University of Medical Sciences, Tabriz, Iran \\ ${ }^{2}$ Department of Epidemiology, Tabriz University of Medical Sciences, Tabriz, Iran \\ ${ }^{3}$ Drug Applied Research Center, Tabriz University of Medical Sciences, Tabriz, Iran \\ "Corresponding author: Drug Applied Research Center, Tabriz University of Medical Sciences, Tabriz, Iran. Email: farshiar@yahoo.com

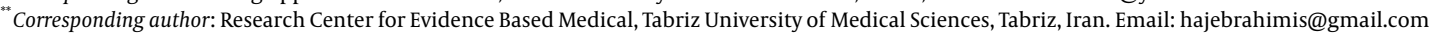 \\ Received 2018 October 19; Accepted 2018 December 03.
}

\begin{abstract}
Objectives: To determine whether tadalafil, a PDE5i, is effective on stent-associated symptoms.

Methods: In this double-blind randomized clinical trial, 80 consecutive male patients with unilateral double J stents were randomly assigned to intervention and placebo groups ( $\mathrm{n}=40$ per group). The patients received $10 \mathrm{mg}$ of tadalafil or placebo once a day for four weeks. The Ureteral Stent Symptom Questionnaire(USSQ) was selected as the primary outcome assessment tool. Complications were recorded as secondary outcomes. Data were analyzed using STATA 11 statistical software. Relative risk (RR) and risk difference (RD) were calculated for appropriately dichotomized endpoints.

Results: Of the 80 patients who provided consent, 72 patients successfully completed the study. The mean urinary symptom index score was 30.94 in the placebo group and 26.59 in the tadalafil group $(\mathrm{P}=0.0003)$. Stent-related pain was reported by $80 \%$ of the patients in the placebo group and by $67.6 \%$ of the patients in the tadalafil group. The mean pain index score was 20.77 in the placebo group and 16.41 in the tadalafil group $(\mathrm{P}=0.039)$. The mean sexual matters score in the placebo and tadalafil groups was 6.20 and 3.73 , respectively $(\mathrm{P}=0.0007)$. There was no significant difference in other domains between the groups. The tadalafil group showed no increase in major side effects compared to the placebo group.

Conclusions: Tadalafil improved stent-related urinary symptoms, pain, sexual status, and overall score; it can also be used as a novel treatment modality in the alleviation of lower urinary tract symptoms and improve the quality of life in these patients.
\end{abstract}

Keywords: Ureteral Stent, Lower Urinary Tract Symptoms, Tadalafil

\section{Background}

Double-J ureteral stent is employed in several conditions including obstructive uropathy, intractable acute renal colic, iatrogenic ureteral injuries, and blunt or penetrating trauma (1). Although these stents play an essential role in urological procedures, the potential side effects and patient morbidities associated with them have been identified as major health concerns (2). Lower urinary tract symptoms (LUTS) such as frequency, urgency, dysuria, and pain occur in most patients (3). More than $80 \%$ of patients experience stent-related pain affecting daily activities, 32\% report sexual problems, and 58\% show reduced work performance (4-6). These symptoms cause a significant decline in health-related quality of life in $45 \%-80 \%$ of patients (7).

Several medical modalities have been applied for stent- associated symptoms relief. Alpha-1 blockers and antimuscarinic agents have been studied in some experiments (810). Alpha-1 blockers had beneficial effects in reducing stent symptoms and pain $(11,12)$. A recent systematic review indicated that anticholinergic agents had slight superiority over placebo (13). On the other hand, the long-term effects of anticholinergic agents were comparable to those of placebo. Furthermore, antimuscarinics have adverse side effects including dry mouth, constipation, blurred vision, somnolence, dizziness, and cognitive impairment (14). Side effects are also associated with alpha-1 blockers and include asthenia, dizziness, and hypotension. Anticholinergics and selective alpha-1 blockers have positive effects on urinary symptoms and pain, but these drugs do not improve patients' sex life (8-10).

Epidemiological studies have indicated the relationship between LUTS and erectile dysfunction (ED). This asso- 
ciation is more than mere coincidence; in fact, it is a causal relationship (15). Multiple studies described LUTS alleviation in patients treated for ED with phosphodiesterase type 5 inhibitors (PDE5 inhibitors) $(16,17)$. Tadalafil is one of these therapeutic options that is widely applied in treating ED $(18,19)$. In addition, the use of tadalafil showed significant clinical improvement in LUTS and benign prostatic obstruction (20).

\section{Objectives}

This study was designed to evaluate the effects of tadalafil compared to placebo on stent-related symptoms improvement based on the Ureteral Stents Symptom Questionnaire (USSQ).

\section{Methods}

This randomized controlled double-blind study was conducted in a university hospital. Subject recruitment began in September 2016. A total of 80 male patients aged 15 70 years with an indication for unilateral ureteral stent insertion were enrolled in the study. The participants were randomly allocated at a 1:1 ratio into placebo and tadalafil groups by computer-generated random blocks. Patients with a history of hypotension or hypertension, cardiac or pulmonary problems, stroke, renal or hepatic failure, retinitis pigmentosa, severe headache, consumption of nitrate drugs, and a positive urine culture or those who showed any allergic reaction to tadalafil were excluded from this trial (Figure 1 ).

The patients enrolled in this study underwent ureteral stent placement. For this study, we used $4.8 \mathrm{~F}, 28 \mathrm{~cm}$ double-J ureteral stents. On the first post-operative day, the medical intervention began. Each patient received placebo or tadalafil tablets (10 mg) orally once a day by a nurse who was unaware of the study's aim. Patients in both groups took routine prophylactic antibiotics, and all the study participants received treatment for 28 consecutive days.

Urinary symptoms, body pain, general health, sexual matters, and work performance were assessed by the Persian version of USSQ (21). Scores for the subscales and the global scores were compared based on groups (control or experimental). All the side effects were recorded in both groups.

Data were analyzed using STATA version 11. Change in the global score of USSQ, in addition to scores from each section, were compared between the groups using either parametric or nonparametric tests considering the distribution of the data. Relative risk (RR) and number needed to treat (NNT) along with their 95\% confidence intervals
(CI) were calculated for appropriately dichotomized endpoints. The principal analysis was on an intention-to-treat basis. P-value less than 0.05 was considered significant.

\section{Results}

Of the 40 patients in the placebo group, five patients were excluded since two of them chose to continue their treatment in private hospitals and three patients who did not have any major complications were reluctant to continue the study. Of the 40 patients in the tadalafil group, three patients were excluded. Two patients discontinued the study by personal desire and the third one had history of panic disorder and his physician advised to discontinue the pills. The mean age of the patients in the placebo and tadalafil groups was 42.3 and 43.3 years, respectively. In the placebo and tadalafil groups, serum creatinine level was 1.43 and 1.48, respectively, and urea level was 34 and 36.5, respectively.

\subsection{Urinary Symptoms}

The mean urgency score was 3.40 in the placebo group and 2.76 in the tadalafil group; dysuria score was 3.23 in the placebo group and 2.65 in the tadalafil group; frequency score was 3.03 in the placebo group and 2.65 in the tadalafil group; nocturia score was 2.40 in the placebo group and 2.62 in the tadalafil group; hematuria score was 2.80 in the placebo group and 2.03 in the tadalafil group; incomplete emptying score was 2.74 in the placebo group and 2.05 in the tadalafil group; urge incontinence score was 1.43 in both groups; incontinence score was 1.17 in the placebo group and 1.03 in the tadalafil group; and the mean score for spending the rest of life with stents, if any, was 5.01 (mostly dissatisfied).

\subsection{Body Pain}

A total of $28(80 \%)$ patients in the placebo group and 25 (67.6\%) patients in the tadalafil group reported pain. In the placebo group, flank, suprapubic, and genitalia pain was common (22.8\%); flank, suprapubic, and inguinal pain was detected in $21.6 \%, 18.9 \%$, and $16.2 \%$ of the tadalafil group patients, respectively; genital pain was reported in $5.7 \%$ of the placebo group patients and in $8.1 \%$ of the tadalafil group patients; abdominal pain was reported in $5.7 \%$ of the placebo group patients and $2.7 \%$ of the tadalafil group. The severity of pain in the placebo and tadalafil group patients was 4.85 and 4.08 , respectively $(\mathrm{P}=0.02)$. In the placebo group, 25 of 35 (71\%) patients and in the tadalafil group, 18 of 37 (49\%) patients required painkillers; however, in patients who took analgesics, drug consumption was similar in both groups (score of 2.8 vs. 2.7). 


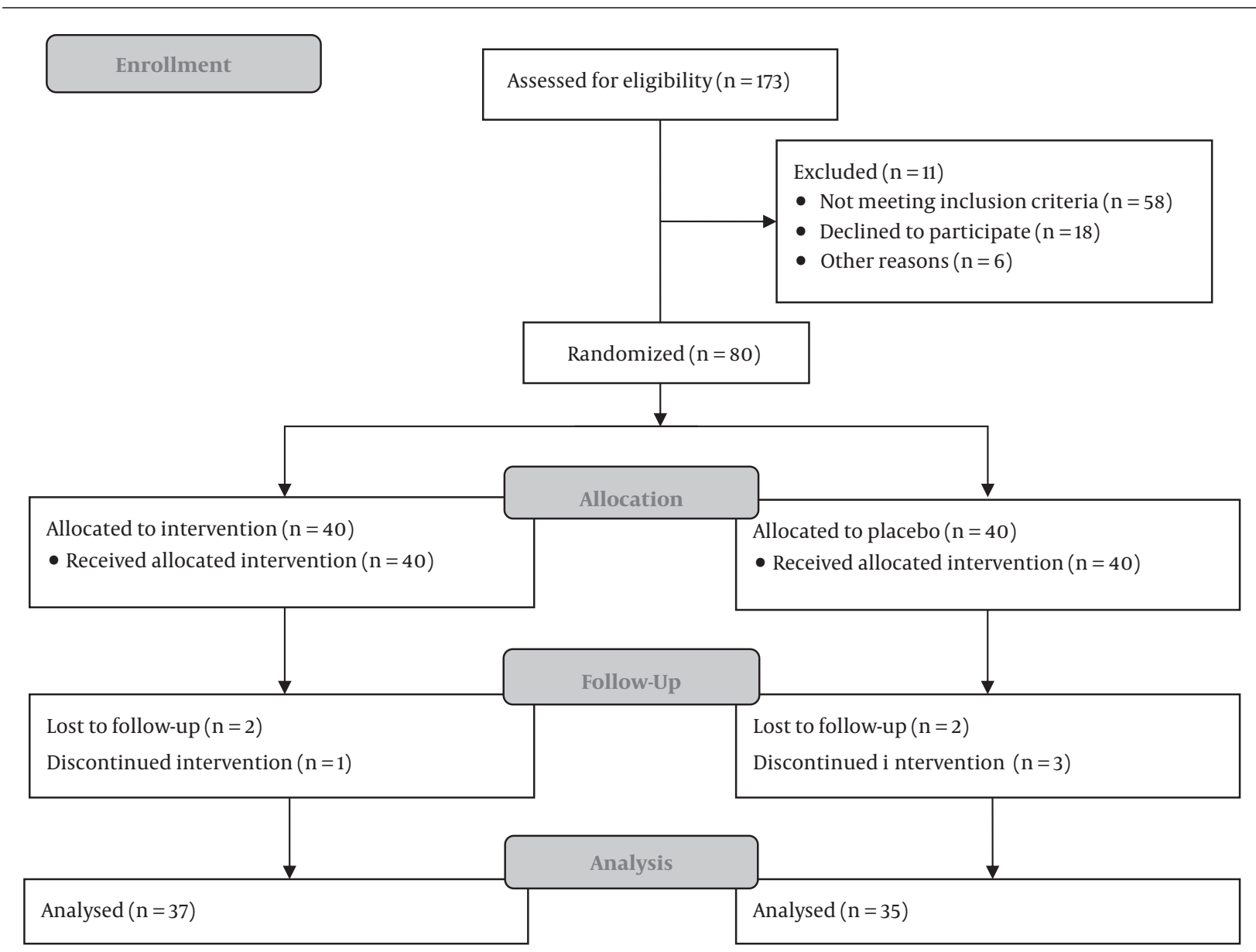

Figure 1. Consort flow diagram

\subsection{General Health}

Feeling calm score was 3.29 and 2.46 in the placebo and tadalafil groups, respectively; feeling tired score was 2.54 and 2.43 in the placebo and tadalafil groups, respectively; enjoying social life score was 2.77 in the placebo group and 2.14 in the tadalafil group; difficulty in heavy physical activity score was 2.11 in the placebo group and 2.19 in the tadalafil group; needing extra help score was 1.74 in the placebo group and 1.95 in the tadalafil group; and difficulty in light physical activities score was 1.74 in the placebo group and 1.68 in the tadalafil group.

\subsection{Work Performance}

Patients were in bed rest for 2.91 days in the placebo group and for 2.43 days in the tadalafil group; patients cut down their routine activities for 5.5 days in the placebo group and for 4.11 days in the tadalafil group; taking frequent rests was reported in $80 \%$ of the placebo group and
$78.4 \%$ of the tadalafil group; working with some changes was reported in $74.3 \%$ of the placebo group and $81.1 \%$ of the tadalafil group; and regular working was acknowledged in only $17.1 \%$ of the placebo group and $16.2 \%$ of the tadalafil group.

\subsection{Sexual Matter}

A total of 42 patients had an active sex life during the study (23 patients in the placebo group and 19 patients in the tadalafil group), of these $47.8 \%$ in the placebo group and $36.8 \%$ in the tadalafil group had pain during intercourse; in addition, of these patients, $26.1 \%$ in the placebo group and $57.9 \%$ in the tadalafil group were very satisfied with their sexual life.

A total of 30 patients had no active sexual relations during the study (12 in the placebo group and 18 patients in the tadalafil group); and of these patients, 9 in the placebo group and 5 patients in the tadalafil group stopped their 
sex life after stent insertion. In addition, of these patients, seven patients in the placebo group and two patients in the tadalafil group stopped their sexual relations because of the stent-associated problems.

\subsection{Additional Problems}

A total of 19 patients in the placebo group and 21 patients in the tadalafil group needed to take antibiotics and took approximately one course of therapy. A total of 15 patients in the placebo group and 16 patients in the tadalafil group did not need to seek help from a health professional; $45.7 \%$ of the placebo group and $40.5 \%$ of the tadalafil group patients visited the hospital for stent-related symptoms.

Tadalafil significantly improved ureteral stent-related symptoms based on the total score of USSQ (RR 1.6, 95\% CI: 1.01 to 2.59$)(\mathrm{NNT}=4.02,95 \% \mathrm{CI}: 2.1$ to 40$)$. As three cases in the tadalafil group and five patients in the placebo group were lost to follow-up, we also conducted an intent to treat analysis based on permutation on total scores in each group (Table 1). The results of intention to treat analysis were totally similar to per-protocol analysis such that the mean difference was calculated to be 98.25 in intention to treat analysis compared to 98.08 in per-protocol analysis. The P-values were 0.0019 and 0.0049 respectively for the intention to treat and per-protocol analyses.

\subsection{Side Effects}

Potential side effects of tadalafil including flushing, hypotension, chest pain, headache, insomnia, asthenia, vertigo, blurred vision, dyspepsia, nausea, diarrhea, gastroesophageal reflux, constipation, myalgia, back pain, arthralgia, pharyngitis, nasal congestion, cough, sinusitis and flulike syndrome were considered as secondary outcomes observed in this study (Table 2). There was no case of priapism in neither group. However, two patients in the tadalafil group had prolonged erection that persisted 1-2 hours, and detumescence occurred without any medication or intervention.

\section{Discussion}

The widespread use of ureteral stents in urologic procedures, especially endourological practice, has been used for several co-morbid conditions including urinary problems, body pain, general health problems, reduced work performance, and deteriorated sexual life due to stent (5). Several studies have been performed for decreasing unpleasant symptoms. Selective alpha-1 blockers as well as anticholinergic and antimuscarinic agents were evaluated in different trials and meta-analyses $(10,13,22,23)$. These treatment modalities have some side effects and affect sexual life. Considering that the majority of stent patients are young and have active sexual relations, impact on sexual life is important. Tadalafil is extensively used for ED and has shown improvements in both LUTS and ED in men with significant problems in both areas without any substantial increase in side effect profile $(20,24)$.

As shown in this study, the use of tadalafil significantly alleviated stent-related urinary symptoms in all subscales. Urgency was the most common symptom (all of placebo group and $89.2 \%$ of the tadalafil group patients) and incontinence was the least common problem $(14.2 \%$ in the placebo group and $2.7 \%$ in the tadalafil). In the present study, tadalafil significantly reduced the prevalence of urinary symptoms.

Eighty percent of the placebo group patients suffered from pain that is in accordance with the study by Joshi et al. (4). Feeling of pain and its severity in the patients receiving tadalafil were significantly less than those in the placebo group. In this group of patients, frequency, urgency, pain during voiding, impaired sexual function, and general health index scores apparently improved. Patients in the tadalafil group were more capable of performing heavy activities and had less pain during intercourse, increasing their overall satisfaction compared to the placebo group. Tadalafil not only reduced pain during voiding, but also diminished genital pain probably with impact on PDE receptors on bladder neck and trigone. Tadalafil also improved sexual performance status in such patients in all subscales. Sexual relations satisfaction in the tadalafil group was significantly higher than the placebo group ( $\mathrm{P}$ $=0.03$ ). These results were expected because tadalafil has been essentially produced for sexual problems.

The general health index score in the tadalafil group was higher than that in the placebo group, but no significant difference was observed between the two groups. Urinary tract infections, need for medical assistance, or even hospitalization rate were lower in the tadalafil group, but the difference was not significant.

Madani et al. in their study on BPH patients demonstrated that tadalafil improves the quality of life and urinary symptoms in patients with LUTS in comparison with placebo (25). Beddingfield et al. in a trial on 55 patients that was methodologically similar to our study reported that patients in alfuzosin arm reported less overall pain. Men in the alfuzosin arm also reported a lower incidence of excessive urination $(\mathrm{P}=0.040)(26)$.

Park et al. compared the effect of alfuzosin, tolterodine ER and placebo on 52 patients with ureteral stents. Urinary symptoms in alfuzosin and toltrodine groups decreased significantly $(\mathrm{P}=0.032)$. Body pain scores were $8.2,11.7$, and 16.2 in alfuzosin, toltrodine, and placebo arms, respectively 


\begin{tabular}{|c|c|c|c|}
\hline & Placebo Group $(\mathrm{N}=35)$ & Tadalafil Group $(N=37)$ & P Value \\
\hline Urinary symptoms score & $30.94 \pm 5.00$ & $26.59 \pm 5.35$ & 0.00 \\
\hline Body pain score & $20.77 \pm 10.04$ & $16.41 \pm 10.70$ & 0.03 \\
\hline General health score & $14.20 \pm 4.06$ & $12.84 \pm 4.46$ & 0.09 \\
\hline Sexual matters score & $6.20 \pm 3.39$ & $3.73 \pm 2.86$ & 0.00 \\
\hline Other problems score & $12.51 \pm 2.73$ & $11.51 \pm 2.79$ & 0.06 \\
\hline Total score & 105.6 & 91 & 0.00 \\
\hline
\end{tabular}

\begin{tabular}{lccc}
\hline Table 2. Side Effects Observed in This Study & & \\
\hline Side Effects & Placebo Group & Tadalafil Group & P Value \\
\hline Cardiovascular & 4 & 9 & 0.92 \\
$\begin{array}{l}\text { Central nervous } \\
\text { system }\end{array}$ & 12 & 14 & 0.73 \\
\hline Gastrointestinal & 14 & 16 & 0.62 \\
\hline Musculoskeletal & 11 & 16 & 0.64 \\
\hline Respiratory & 6 & 8 & 0.47 \\
\hline Genitourinary & 0 & 2 & 0.16 \\
\hline
\end{tabular}

\subsection{Conclusions}

This is the first trial to study the effect of tadalafil, as a novel drug, on stent-associated symptoms, and according to the results tadalafil reduced the adverse stent-associated symptoms and improved the quality of life in patients with ureteral stents. Additionally, the results confirmed the efficacy and safety of tadalafil in these patients, which can open a new window to improve stent-related symptoms.

\section{Footnotes}

$(\mathrm{P}=0.020)$. There were no significant differences in urinary symptoms and pain between the alfuzosin and tolterodine groups. In addition, there was no significant difference in the general health, work performance, and sexual performance scores among the groups (8). In our study, urinary symptoms and pain scores improved similarly, albeit sexual function in our study had more improvement than this trial.

According to the findings of the aforementioned studies, most of the medications used for alleviating stentassociated symptoms were only effective on urinary symptoms and pain; however, in our study, sexual problems were improved significantly more than the mentioned studies; also, other problems had decreased in the tadalafil arm, but not in a significant manner $(\mathrm{P}=0.06)$.

All of the above studies failed to show improvement in work performance as in our study. There were no significant probable tadalafil-associated side effects including priapism, headache, dyspepsia, facial flushing, backache, myalgia, blurred/blue vision, and hypotension in the patients.

The limitation of our study was that female patients were not enrolled as the effects of tadalafil on females are not well-established.

Authors' Contribution: Study concept and design: Alireza Farshi Haghro and Sakineh Hajebrahimi. Analysis and interpretation of data: Sakineh Hajebrahimi and Hossein Babaei. Drafting of the manuscript: Alireza Farshi Haghro, Abbas Jabbari and Hossein Babaei. Revision of the manuscript: Alireza Farshi Haghro, Sakineh Hajebrahimi, and Hossein Babaei. Statistical analysis: Homayoun Sadeghi.

Conflict of Interests: There are no financial interests related to the material of the manuscript.

Ethical Considerations: This trial was approved by Ethics Committee of Tabriz University of Medical Sciences (9291) and was registered at the Clinical Trial Registry (IRCT2013113015597N1).

Funding/Support: This study was performed by the financial support of Drug Applied Research Center, Tabriz University of Medical Sciences, Iran.

Patients Consent: All the patients provided informed consent.

\section{References}

1. Jeong H, Kwak C, Lee SE. Ureteric stenting after ureteroscopy for ureteric stones: A prospective randomized study assessing symptoms and complications. BJU Int. 2004;93(7):1032-4. discussion 1034-5. doi: 10.1111/j.1464-410X.2004.4776a.x. [PubMed: 15142158]. 
2. Irani J, Siquier J, Pires C, Lefebvre O, Dore B, Aubert J. Symptom characteristics and the development of tolerance with time in patients with indwelling double-pigtail ureteric stents. BJUInt.1999;84(3):2769. doi:10.1046/j.1464-410x.1999.00154.x. [PubMed: 10468721].

3. Haleblian G, Kijvikai K, de la Rosette J, Preminger G. Ureteral stenting and urinary stone management: A systematic review. $J$ Urol. 2008;179(2):424-30. doi: 10.1016/j.juro.2007.09.026. [PubMed: 18076928].

4. Joshi HB, Stainthorpe A, MacDonagh RP, Keeley FX Jr, Timoney AG, Barry MJ. Indwelling ureteral stents: Evaluation of symptoms, quality of life and utility. J Urol. 2003;169(3):1065-9. discussion 1069. doi: 10.1097/01.ju.0000048980.33855.90. [PubMed: 12576847].

5. Joshi HB, Okeke A, Newns N, Keeley FX Jr, Timoney AG. Characterization of urinary symptoms in patients with ureteral stents. Urology. 2002;59(4):511-6. doi: 10.1016/S0090-4295(01)01644-2. [PubMed: 11927301]

6. Mokhtari G, Shakiba M, Ghodsi S, Farzan A, Heidari Nejad S, Esmaeili S Effect of terazosin on lower urinary tract symptoms and pain due to double-J stent: A double-blind placebo-controlled randomized clinical trial. Urol Int. 2011;87(1):19-22. doi: 10.1159/000323855. [PubMed: 21597261].

7. Yakoubi R, Lemdani M, Monga M, Villers A, Koenig P. Is there a role for $\alpha$-blockers in ureteral stent related symptoms? A systematic review and meta-analysis. J Urol. 2011;186(3):928-34. doi: 10.1016/j.juro.2011.04.061.

8. Park SC, Jung SW, Lee JW, Rim JS. The effects of tolterodine extended release and alfuzosin for the treatment of double-j stent-related symptoms. J Endourol. 2009;23(11):1913-7. doi: 10.1089/end.2009.0173. [PubMed: 19814699].

9. Wang CJ, Huang SW, Chang $\mathrm{CH}$. Effects of specific alpha-1A/1D blocker on lower urinary tract symptoms due to double-J stent: A prospectively randomized study. Urol Res. 2009;37(3):147-52. doi: 10.1007/s00240-009-0182-8. [PubMed: 19277623].

10. Tehranchi A, Rezaei Y, Khalkhali HR, Rezaei M. Effects of terazosin and tolterodine on ureteral stent related symptoms: A doubleblind placebo-controlled randomized clinical trial. Int Braz J Urol. 2013;39(6):832-40. doi:10.1590/s1677-5538.ibju.2013.06.09.

11. Deliveliotis C, Chrisofos M, Gougousis E, Papatsoris A, Dellis A, Varkarakis IM. Is there a role for alpha1-blockers in treating double-J stent-related symptoms? Urology. 2006;67(1):35-9. doi: 10.1016/j.urology.2005.07.038. [PubMed:16413328].

12. Damiano R, Autorino R, De Sio M, Giacobbe A, Palumbo IM, D'Armiento M. Effect of tamsulosin in preventing ureteral stentrelated morbidity: A prospective study. J Endourol. 2008;22(4):651-6. doi: 10.1089/end.2007.0257. [PubMed: 18338955].

13. Nabi G, Cody JD, Ellis G, Hay-Smith J, Herbison GP. Anticholinergic drugs versus placebo for overactive bladder syndrome in adults. Cochrane Database Syst Rev. 2006;(4). doi: 10.1002/14651858.CD003781.pub2.

14. Patel B, Bavendam T, Badlani G. Use of antimuscarinics in the el- derly. ScientificWorldJournal. 2009;9:459-65. doi: 10.1100/tsw.2009.55 [PubMed: 19526185]. [PubMed Central: PMC5823097].

15. Speakman MJ. PDE5 inhibitors in the treatment of LUTS. Curr Pharm Des. 2009;15(30):3502-5. doi: 10.2174/138161209789207051. [PubMed: 19860696].

16. Oger S, Behr-Roussel D, Gorny D, Lebret T, Validire P, Cathelineau X, et al. Signalling pathways involved in sildenafil-induced relaxation of human bladder dome smooth muscle. Brit J Pharm. 2010;160(5):113543. doi: 10.1111/j.1476-5381.2010.00748.x.

17. Bittencourt JA, Tano T, Gajar SA, Resende AC, de Lemos Neto M, Damiao $\mathrm{R}$, et al. Relaxant effects of sildenafil on the human isolated bladder neck. Urology. 2009;73(2):427-30. doi: 10.1016/j.urology.2008.06.060. [PubMed: 18774591].

18. Carson CC, Rajfer J, Eardley I, Carrier S, Denne JS, Walker DJ, et al. The efficacy and safety of tadalafil: An update. BJU Int. 2004;93(9):1276-81. doi:10.1111/j.1464-410X.2004.04819.x. [PubMed: 15180622].

19. Forgue ST, Patterson BE, Bedding AW, Payne CD, Phillips DL, Wrishko RE, et al. Tadalafil pharmacokinetics in healthy subjects. $\mathrm{Br} J$ Clin Pharmacol. 2006;61(3):280-8. doi: 10.1111/j.1365-2125.2005.02553.x. [PubMed: 16487221]. [PubMed Central: PMC1885023].

20. McVary KT, Roehrborn CG, Kaminetsky JC, Auerbach SM, Wachs B Young JM, et al. Tadalafil relieves lower urinary tract symptoms secondary to benign prostatic hyperplasia. JUrol. 2007;177(4):1401-7. doi: 10.1016/j.juro.2006.11.037. [PubMed:17382741].

21. Jabbari A, Farshi A, Sadeghi Bazargani H, Hajebrahimi S. Validity and reliability of the ureteral stent symptoms questionnaire to Persian language. J Anal Res Clin Med. 2015;3(1):17-22. doi: 10.15171/jarcm.2015.003.

22. Lim KT, Kim YT, Lee TY, Park SY. Effects of tamsulosin, solifenacin, and combination therapy for the treatment of ureteral stent related discomforts. Korean J Urol. 2011;52(7):485-8. doi: 10.4111/kju.2011.52.7.485. [PubMed: 21860770]. [PubMed Central: PMC3151637].

23. Lee YJ, Huang KH, Yang HJ, Chang HC, Chen J, Yang TK. Solifenacin improves double-J stent-related symptoms in both genders following uncomplicated ureteroscopic lithotripsy. Urolithiasis. 2013;41(3):24752. doi: 10.1007/s00240-013-0554-y. [PubMed: 23515684].

24. Kohler TS, McVary KT. The relationship between erectile dysfunction and lower urinary tract symptoms and the role of phosphodiesterase type 5 inhibitors. Eur Urol. 2009;55(1):38-48. doi: 10.1016/j.eururo.2008.08.062. [PubMed:18783872].

25. Madani AH, Afsharimoghaddam A, Roushani A, Farzan A, Asadollahzade A, Shakiba M. Evaluation of tadalafil effect on lower urinary tract symptoms of benign prostatic hyperplasia in patients treated with standard medication. Int Braz J Urol. 2012;38(1):33-9. doi: 10.1590/S1677-55382012000100005. [PubMed: 22397784].

26. Beddingfield R, Pedro RN, Hinck B, Kreidberg C, Feia K, Monga M. Alfuzosin to relieve ureteral stent discomfort: A prospective, randomized, placebo controlled study. J Urol. 2009;181(1):170-6. doi: 10.1016/j.juro.2008.09.026. [PubMed: 19013590]. 